\title{
La depresión en la tercera edad Resumen del Consenso del Instituto Nacional de la Salud, EE.UU.
}

Consensus Statement:Diagnosis and Treatment of depression in Late Life. Consensus Statement Update. Lebowitz BD, Pearson JL, Schneider LS y cols. JAMA 1997;278:1186-1190

\section{Introducción}

Un grupo de expertos decidió revisar las conclusiones del Consenso de 1991 organizado por el Instituto Nacional de la Salud (NIH) de EE.UU. Dicho Consenso trata acerca del diagnóstico y tratamiento de la depresión en la tercera edad basándose en la evidencia científica que hay hasta el momento. La revisión concluyó que, a pesar de que el consenso inicial se mantiene, hay importantes novedades en algunas áreas. Estas áreas incluyen el comienzo y el curso de la depresión en la tercera edad, comorbilidad y discapacidad, sexo y hormonas, nuevos medicamentos, psicoterapia y enfoque de tratamientos crónicos, impacto de la depresión en los servicios de salud y utilización de recursos, depresión en la tercera edad como factor de riesgo para el suicidio y la importancia de la heterogeneidad de las formas de depresión.

El Panel del Consenso concluyó que la enfermedad depresión es frecuente entre los ancianos, que la depresión en la tercera edad es un serio problema de salud pública, y que la comorbilidad de la depresión en esta edad es particularmente problemática. Además se concluyó que la depresión puede ser diagnosticada y diferenciada de lo que sería una forma normal del envejecimiento, que hay una variedad de tratamientos que han demostrado ser seguros y eficaces en los ancianos, y que la depresión puede ser una enfermedad recurrente y que por lo tanto el tratamiento de larga duración puede estar indicado.

\section{Comienzo y curso de la depresión}

Investigaciones recientes han descubierto heterogeneidad clínica y biológica de la depresión que comienza en la última etapa de la vida, y diversos subgrupos con patrones de apariencia clínica, mecanismos y resultados diferentes. El comienzo en general es temprano en la vida, con un curso recurrente luego de un primer episodio en la más temprana adultez. El primer episodio también puede ocurrir en la época tardía de la vida. Comparado con pacientes de la misma edad con depresión recurrente, aquellos pacientes que tuvieron su primer episodio en la tercera edad son más propensos a tener un curso más crónico. El comienzo a edades más tardías podría a veces estar asociado con una variedad de anormalidades cerebrales, tales como dilatación ventricular, trastornos en la sustancia blanca o deterioro cognitivo.

En este grupo etario la depresión con deterioro cognitivo suele ser en algunos casos predictor de una demencia, tal como el Alzheimer o la demencia vascular, a pesar de que pueda revertirse con tratamiento antidepresivo .

La depresión en geriatría es a menudo una enfermedad comórbida junto a desórdenes vasculares y otras patologías.

\footnotetext{
Comorbilidad y discapacidad

La depresión coexiste con enfermedades físicas que han mostrado aumentar los niveles de discapacidad funcional, el uso de recursos en salud, y reducir la efectividad de la rehabilitación en los pacientes más añosos. En los más ancianos con deterioro de la visión, la depresión está más fuertemente asociada con la discapacidad que con el grado de pérdida de la visión o con la sobrecarga médica.
}

\section{La depresión como subsindrome del anciano}

Mientras recientes estudios reportan un $1 \%$ a $2 \%$ de prevalencia de la depresión mayor, y un $2 \%$ de distimia entre los ancianos de la comunidad, una frecuencia mucho mayor se ha visto con el subsindrome depresivo (13\% a 27\%). El subsíndrome depresivo se refiere a síntomas de depresión que están asociados a un aumento del riesgo de depresión mayor, discapacidad física, enfermedades médicas y alto uso de los servicios de salud. Estos síntomas no alcanzan a cumplir los criterios diagnósticos del DSM IV para depresión mayor o la distimia. En pacientes con enfermedades médicas y en pacientes institucionalizados en geriátricos la prevalencia de tales síntomas son de aproximadamente un 50\%. El curso del subsindrome es variable, pero en el contexto de severa discapacidad física, por ej. por accidente cerebrovascular, los síntomas de depresión pueden extenderse por largos períodos. Típicamente los síntomas de depresión de los más ancianos no son encontrados por los que proveen los cuidados. Los médicos han comenzado a identificar y tratar el subsindrome depresivo a partir de reconocer el innecesario sufrimiento humano y la carga que implica al sistema de salud.

Investigaciones iniciales han sugerido que la psicoterapia corta es factible, aceptable y efectiva en la reducción de síntomas depresivos a corto plazo en los ancianos enfermos. Otros estudios que se están llevando a cabo investigan sobre la medicación antidepresiva (ISRS, inhibidores selectivos de la recaptación de serotonina) y psicoterapias (interpersonales o de resolución de problemas) en los ancianos. Los resultados de estos estudios proveerán importantes datos acerca del curso clínico y del tratamiento del subsindrome depresivo. Con el tiempo, la identificación, evaluación clínica y monitoreo del subsindrome depresivo como indicador de riesgo para depresión según escalas validadas, será requerido como técnica de rastreo.

\section{Sexo y hormonas}

Uno de los hallazgos más consistentes dentro de la epidemiología de la psiquiatría es que la mujer padece, con mayor frecuencia que los hombres, todos los tipos de depresión. El aumento de depresión se ha observado en las mujeres en la evolución de la menopausia ya sea natural o quirúrgica y en respuesta a la terapia antiestrogénica del cáncer de mama. Los síntomas atribuidos a la menopausia incluyen ánimo depresivo, disminución de la confianza en sí misma, dificultad en tomar decisiones, ansiedad, insomnio, fatiga, problemas en la memoria y en la concentración y disminución de la libido. El reemplazo hormonal se ha asociado con la mejoría en la calidad de vida, pero estos datos son aún preliminares. No hay evidencia que sostenga los efectos antidepresivos de los andrógenos, como la tetosterona o la dehidroespiandosterona, tanto en el hombre como en la mujer.

La relación de factores hormonales en el desarrollo y tratamiento de la depresión está aun poco definida. Para determinar si el tratamiento con estrógenos y esteroides tiene un rol significativo en el tratamiento de la depresión en la tercera edad, serán necesarios ensayos clínicos prospectivos. 


\section{¿Qué es un tratamiento seguro para la depresión en la vejez? ¿Cuáles son las indicaciones y las contraindicaciones para un tratamiento específico?}

El objetivo del tratamiento es aliviar los síntomas, prevenir la recurrencia, y mejorar la calidad de vida y la funcionalidad del paciente. En la conferencia de 1991, la fluoxetina, el primer ISRS, ya había sido comercializada durante 2 años, y existían pocos datos de su utilidad en los ancianos. Las conclusiones del panel previo acerca de la farmacoterapia, se basaron largamente sobre investigaciones de los antidepresivos tricíclicos.

Un número de otros compuestos antidepresivos han sido introducidos desde 1990, incluyendo los ISRS sertralina y paroxetina, y drogas de acción mixta incluyendo la ven lafaxina, nefazodona y la mirazapina. Estas nuevas drogas han sido vistas como un potencial progreso para el tratamiento de los ancianos por su menor cantidad de efectos anticolinérgicos y cardiovasculares indeseables que están asociados a los tricíclicos.

\section{Eficacia}

En ensayos que directamente comparan los ISRS con ATCs (antidepresivos tricíclicos) la fluoxetina, sertralina y paroxetina son ampliamente equivalentes en eficacia a los ATCs con $60 \%$ a $80 \%$ de respuesta de los pacientes al tratamiento. Los datos de la eficacia no son tan contundentes cuando se compara ISRS con placebo. El único reporte disponible de un estudio randomizado en pacientes ancianos ambulatorios de fluoxetina vs. placebo encontró una menor eficacia que la que se encontró en otros estudios con ATCs. Hay datos limitados acerca de la velafaxina, nefazodone y mitrazapina en pacientes ancianos y aún no es posible sacar conclusiones.

\section{Efectos adversos}

Al igual que los jóvenes, los ancianos tienden a tolerar mejor los ISRS que Los ATCs. Estos hallazgos se basan en menores efectos anticolinérgicos y mínimos efectos cardiovasculares. Efectos comunes de los ISRS incluyen náuseas, diarrea, insomnio, cefalea, agitación y ansiedad. Los efectos adversos de los ISRS que pueden ser más problemáticos en los ancianos incluyen el sindrome de secreción inapropiada de hormona antidiurética, síntomas extrapiramidales, y bradicardia.

Los ATCs varían con respecto al tipo de efectos adversos, pero algunos tales como hipotensión ortostática, sedación, y efectos cardíacos indeseables pueden ser particularmente problemáticos en los ancianos. Se ha sugerido que los ATCs podrían provocar arritmias en pacientes con enfermedad isquémica.

La tasa de abandono de los pacientes con ISRS es de 30 a un $50 \%$ menor que la de los tratados con ATCs.

A pesar de todo los ISRS y los ATCS pueden ser comparables en cuanto a la eficacia en los ancianos, si bien la efectividad de los ISRS puede ser mayor dada la facilidad del uso, menos dosis para ajustar, menor toxicidad y mayor aceptación.

\section{Psicoterapia}

Las investigaciones en la psicoterapia han provisto datos importantes en ciertas áreas donde los paneles identificaron brechas. La conclusión del panel sostiene la eficacia de la psi- coterapia en la tercera edad, a partir de reportes acerca de la utilidad tanto de la psicoterapia aislada como del tratamiento combinado con drogas. Los estudios han demostrado claramente la eficacia de realizar un acercamiento sistematizado al tratamiento, tanto con las técnicas de terapia cognitiva como de terapia interpersonal (no mencionan la técnica psicoanalítica, muy utilizada en nuestro país). La psicoterapia es un componente importante de un tratamiento basado en estrategias de largo plazo donde la contribución de la terapia ha demostrado proveer beneficios sustanciales en prolongar los períodos de buena salud libres de depresión.

\section{Tratamientos de larga duración}

Se ha acumulado evidencia que respalda la necesidad de tratamientos prolongados en la depresión de la tercera edad. Los paneles de 1991 recomiendan el tratamiento por al menos 6 meses luego de la recuperación para aquellos que han tenido un primer episodio y de al menos 12 meses para los que presentan una enfermedad recurrrente. Por cierto, los pacientes ancianos con depresión recurrente pueden necesitar tratamiento antidepresivo indefinidamente para permanecer estables. Además el tratamiento de larga duración sería del mismo tipo, y de la misma intensidad, que el que fue necesario en la primera fase, la fase aguda. Esto contrasta mucho con la práctica habitual. Los seguimientos longitudinales demuestran que la intensidad del tratamiento antidepresivo típicamente disminuye prematuramente, antes de las 8 semanas de la recuperación. La intensidad apropiada de la psicoterapia de mantenimiento no ha sido estudiada en profundidad. La respuesta al tratamiento de larga duración en los ancianos es generalmente similar a la observada en los adultos de mediana edad, pero el riesgo de recaída es mayor.

¿Cuáles son los patrones de uso de servicios de salud en la tercera edad?

Los datos demuestran que longitudinalmente los sintomas de depresión en los ancianos ambulatorios están asociado con un incremento de las visitas al médico y uso de medicación, mayor contidad de visitas a la guardia, y aumento de la carga asistencial. Por todo esto los costos médicos de los pacientes depresivos es significtivamente mayor que el de los no deprimidos.

Recientemente se han analizado datos de 1987 del servicio médico nacional de EE.UU. que mostró que más del $55 \%$ de las personas ancianas que usaron cuidados de salud mental recibierón estos cuidados por el médico de atención primaria. En contraste menos del $3 \%$ de los $>$ de 65 años reportaron haber recibido tratamiento ambulatorio por profesionales de la salud mental, una proporción menor que al de cualquier otro grupo etario.

Los ancianos se verán muy beneficiados si los médicos de atención primaria aumentan su capacidad para detectar y tratar la depresión.

¿Cuáles son los beneficios de reconocer y dar tratamiento adecuado a la depresión en la tercera edad?

¿Cuáles son las consecuencias de no reconocer y de dar tratamiento inadecuado para la misma? 
Estudios recientes han reforzado la asociación de suicidio con depresión mayor, especialmente en los ancianos. En los ancianos con depresión unipolar de comienzo tardío, es característico que se produzcan suicidios. Esto sin embargo es poco probable en las personas que han tenido una correcta evaluación, seguimiento y tratamiento de su depresión.

Desde 1980 a 1992 la frecuencia de suicidios en ancianos aumentó $9 \%$ en EE.UU. Más llamativo fue el aumento del 35\% entre los ancianos de 80 a 84 años. La frecuencia del suicidio entre los ancianos "más ancianos" (aquellos > de 85años) es 6 veces mayor que en la población general e implica un problema de salud pública.

La falla para reconocer y tratar la depresión en aquellos pacientes no fue causada por la restricción del acceso a los cuidados. La mayoría de estas víctimas fueron vistas por un médico de atención primaria en el último mes de sus vidas y el
$39 \%$ en la última semana.

La detección y el tratamiento de la depresión por el médico de atención primaria puede ser mejorada por intervenciones educacionales incluyendo desarrollo y distribución de materiales, lecturas y casos clínicos. Luego de un programa en una ciudad sueca disminuyó la tasa de suicidios en esa ciudad pero no en el resto de Suecia. A pesar de que este estudio no fue controlado resulta alentador. La intervención fue específica para reducir la proporción de depresión relacionada con suicidio; esta disminuyó del $50 \%$ al $12 \%$. Mejoró el tratamiento de la depresión por el médico de atención primaria. Esto se reflejó en que disminuyeron los días de internación de los pacientes internados por depresión y aumentaron las prescripciones de antidepresivos. También hubo una disminución en el uso de los tranquilizantes mayores e hipnóticos, ambos inapropiados para el tratamiento de la depresión.

\section{COMENTARIO}

Queda claro en este artículo y en todos aquellos que se refieran a la depresión en la tercera edad que éste es un problema mayor de salud pública. Esta enfermedad causa mucha morbilidad y también aumenta la mortalidad de los que la padecen. Genera mucho sufrimiento en la gente a la que no se le realizó un adecuado diagnóstico o está subtratada, y genera también pesar en las familias e instituciones que proveen cuidados a los ancianos.

La presente publicación es un resumen de un Consenso de expertos y, como tal, no cumple con una rigurosa metodología explícita de revisión de la literatura. Su intención es, principalmente, actualizar algunas áreas importantes en las cuales se hubieran identificado avances significativos en el manejo y tratamiento de esta enfermedad. La depresión se presenta generalmente con características típicas como ánimo deprimido, pérdida de peso, dificultad en la concentración, llanto frecuente, sentimientos de culpa, ideas de muerte, disturbios en el sueño, pérdida de interés,etc. Los síntomas que produce la depresión en los ancianos suelen ser tan insidiosos que muchas veces los médicos no la pueden reconocer en el contexto de "múltiples problemas físicos". Dada la prevalencia de enfermedades crónicas tan características que este grupo etario padece y los problemas económicos que los afectan no solo en nuestro país, muchos médicos toman como una consecuencia normal la depresión a esta edad, como una actitud que presentan los pacientes. Estos factores conspiran contra el diagnóstico y por ende pueden llevar a un tratamiento incorrecto.
Muchos estudios han demostrado que el tratamiento de la depresión es seguro y efectivo aún en pacientes con mucha comorbilidad agregada y sugieren que el tratamiento de la depresión puede reducir de una manera importante los niveles de discapacidad (1). Esto resultaría en una mejora de los niveles de funcionalidad. La discapacidad funcional en sí misma es un predictor para desarrollar depresión y recíprocamente los síntomas depresivos son un factor de riesgo para el comienzo o la progresión de una discapacidad.

La depresión mayor aumenta la utilización de recursos médicos y aumenta los costos en salud (2). Estudios recientes han reforzado la asociación de suicidio con depresión mayor, especialmente en los ancianos. A pesar de que las enfermedades físicas han sido consideradas como un factor de riesgo para el suicidio en la tercera edad, no hay datos concluyentes recolectados en forma apropiada que sostengan esta conclusión.

La esfera y responsabilidad del médico de atención primaria está siendo expandida y redefinida en el sistema de salud. Cada vez es mayor su responsabilidad en el diagnóstico, tratamiento, y manejo a largo plazo de problemas prevalentes, incluyendo el cuidados de los desórdenes mentales en los pacientes más ancianos.

El reconocimiento temprano de la depresión en los ancianos por parte del médico de familia permite realizar el tratamiento adecuado oportunamente y de esta manera mejorar la calidad de vida, prevenir el sufrimiento que esta enfermedad genera y mantener niveles óptimos de funcionalidad e independencia de los ancianos.

Dra. M. Judith Estremero

Unidad de Medicina Familiar y Preventiva. Hospital Italiano de Buenos Aires.

\section{Referencias}

1. Rothschild AJ. The diagnosis and treatment of late-life depression. J Clin Psychiatry 1996:57 Suppl 5:5-11.

2. Callahan CM, Kesterson JG, Tierney WM. Association of symptoms of depression with diagnostic test charges among older adults. Ann Intern Med. 1997; 126(6): 426-432. 